\title{
Analysis and interpretation of a fast limb CME with eruptive prominence, C-flare, and EUV dimming
}

\author{
S. Koutchmy ${ }^{1}$, V. Slemzin ${ }^{2}$, B. Filippov ${ }^{3}$, J.-C. Noens ${ }^{4}$, D. Romeuf ${ }^{5}$, and L. Golub ${ }^{6}$ \\ 1 Institut d'Astrophysique de Paris, CNRS and Univ. P. \& M. Curie, 98bis boulevard Arago, 75014 Paris, France \\ e-mail: koutchmy@iap.fr \\ 2 P.N. Lebedev Physical Institute, Leninsky pr. 53, Moscow 119991, Russia \\ e-mail: slem@mail1.lebedev.ru \\ 3 Pushkov Institute of Terrestrial Magnetism, Ionosphere and Radio Wave Propagation, Russian Academy of Sciences (IZMIRAN), \\ Troitsk Moscow Region, 142190, Russia \\ e-mail: bfilip@izmiran.troitsk.ru \\ ${ }^{4} \mathrm{OMP}$ and Pic du Midi Observatory, France \\ e-mail: noens@ast.obs-mip.fr \\ 5 C.R.I. Claude Bernard Lyon I University, O.A.-Fiducial, France \\ e-mail: David.Romeuf@recherche.univ-lyon1.fr \\ ${ }^{6}$ Harvard-Smithsonian Center for Astrophysics, 60 Garden Street MS58, Cambridge, MA 02138, USA \\ e-mail: golub@cfa.harvard.edu
}

Received 18 July 2007 / Accepted 7 December 2007

\section{ABSTRACT}

\begin{abstract}
Aims. Coronal mass ejections or CMEs are large dynamical solar-corona events. The mass balance and kinematics of a fast limb $\mathrm{CME}$, including its prominence progenitor and the associated flare, will be compared with computed magnetic structures to look for their origin and effect.

Methods. Multi-wavelength ground-based and spaceborne observations are used to study a fast W-limb CME event of December 2, 2003, taking into account both on and off disk observations. Its erupting prominence is measured at high cadence with the Pic du Midi full $\mathrm{H} \alpha$ line-flux imaging coronagraph. EUV images from SOHO/EIT and CORONAS-F/SPIRIT space instruments are processed including difference imaging. SOHO/LASCO images are used to study the mass excess and motions. Computed coronal structures from extrapolated surface magnetic fields are compared to observations.

Results. A fast bright expanding coronal loop is identified in the region recorded slightly later by GOES as a C7.2 flare, followed by a brightening and an acceleration phase of the erupting material with both cool and hot components. The total coronal radiative flux dropped by 7\% in the $19.5 \mathrm{~nm}$ channel and by $4 \%$ in the $17.5 \mathrm{~nm}$ channel, revealing a large dimming effect at and above the limb over a $2 \mathrm{~h}$ interval. The typical 3-part structure observed $1 \mathrm{~h}$ later by the Lasco $\mathrm{C} 2$ and $\mathrm{C} 3$ coronagraphs shows a core shaped similarly to the eruptive filament/prominence. The total measured mass of the escaping CME $\left(\sim 1.5 \times 10^{16} \mathrm{~g}\right.$ from C2 LASCO observations) definitely exceeds the estimated mass of the escaping cool prominence material although assumptions made to analyze the $\mathrm{H} \alpha$ erupting prominence, as well as the corresponding EUV darkening of the filament observed several days before, made this evaluation uncertain by a factor of 2 . This mass budget suggests that the event is not confined to the eruption region alone. From the current free extrapolation we discuss the shape of the magnetic neutral surface and a possible scenario leading to an instability, including the small scale dynamics inside and around the filament.
\end{abstract}

Key words. Sun: activity - Sun: filaments - Sun: prominences - Sun: coronal mass ejections (CMEs) - Sun: flares

\section{Introduction}

Coronal Mass Ejections (CMEs) were originally defined as large coronal dynamical phenomena (coronal transients) propagating outward in the field of view of white-light (W-L) externally occulted space-borne coronagraphs and often showing three parts (see Wagner 1984). Their primary property is the mass transport of a presumably coronal mass originally situated behind the occulting device, toward the external part, without specifying the origin of this mass revealed by the W-L event due to the scattering on free electrons. Modelers had to address the fundamental question: from where does this large amount of coronal plasma originate? Although many statistical studies of CMEs were published in recent decades (among the latest, see Vourlidas et al. 2000; Cremades \& Bothmer 2004; Gopalswamy 2006) this question has not been systematically considered and no clear answer exists. One possibility is that the mass of the CME comes from a related erupting prominence (EP) and its immediate surroundings. This assumption was seriously considered recently and prompted another question: what is the mass of the EP (see Gilbert et al. 2005, 2006)? However, the general consensus is that only the core of the 3-part CME corresponds to the prominence.

A distinction has been made in considering the presumably more important fast CMEs, usually related to a flare and more likely to be geoeffective and with space weather implications (see e.g. Bao et al. 2007). The EP connection is then sometimes omitted in favor of a discussion of the origin of solar energetic particles that contribute only a little to the mass budget.

CMEs are often associated with erupting filaments or prominences even when considered far from the Sun (see Bothmer \& Schwenn 1994). In the last few years many investigations were 

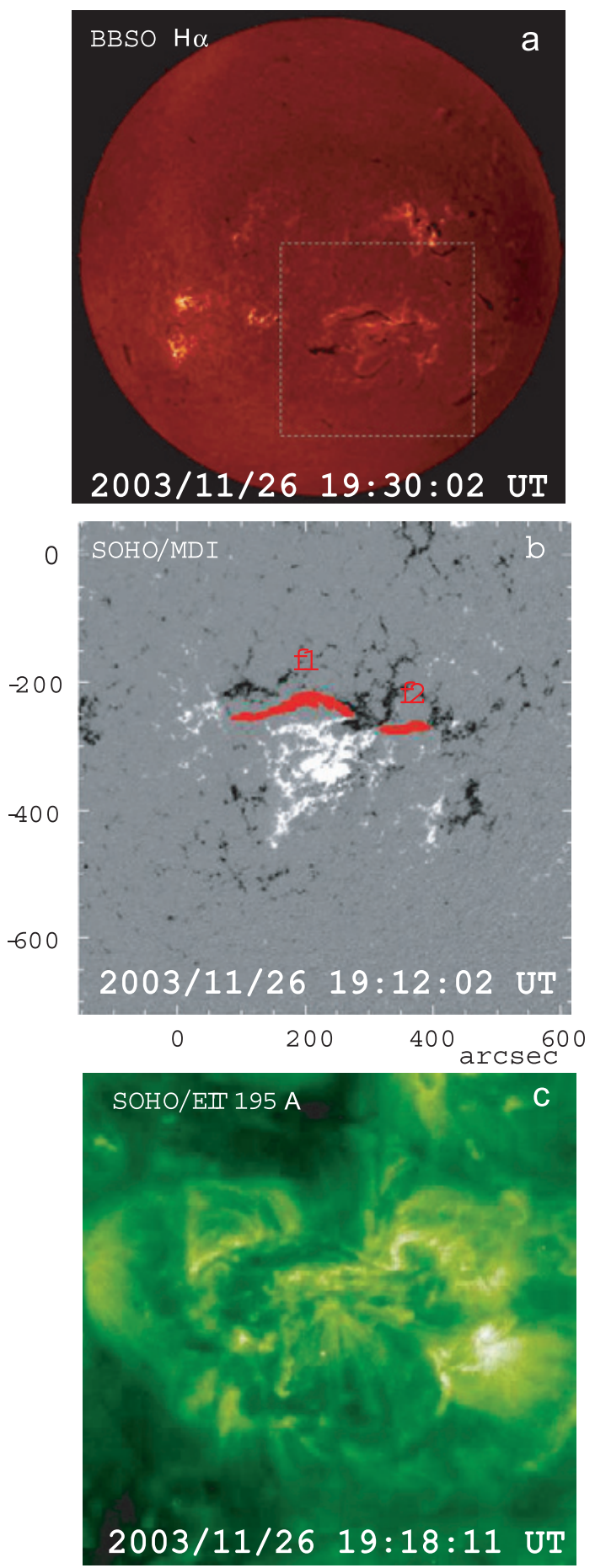

Fig. 1. The BBSO $\mathrm{H} \alpha$ image a) showing the zoom box corresponding to the MDI magnetic map b) of the AR 10508 region with superimposed contours of filaments (red) from the BBSO $\mathrm{H} \alpha$ image; c) EIT image of the box region at $195 \AA$. All images are taken on November 26, 2003.

undertaken to study the relationship between prominence activity and CMEs in different wavelengths. Based on $\mathrm{H} \alpha$ observations at the Mauna Loa Solar Observatory, Gilbert et al. (2000) proposed a classification of active and eruptive prominences according to their kinematical properties (radial height, velocity and acceleration). A separation between escaping prominence material lifting away from the Sun and material returning toward the solar surface would occur in the height range from $1.20 R_{\odot}$

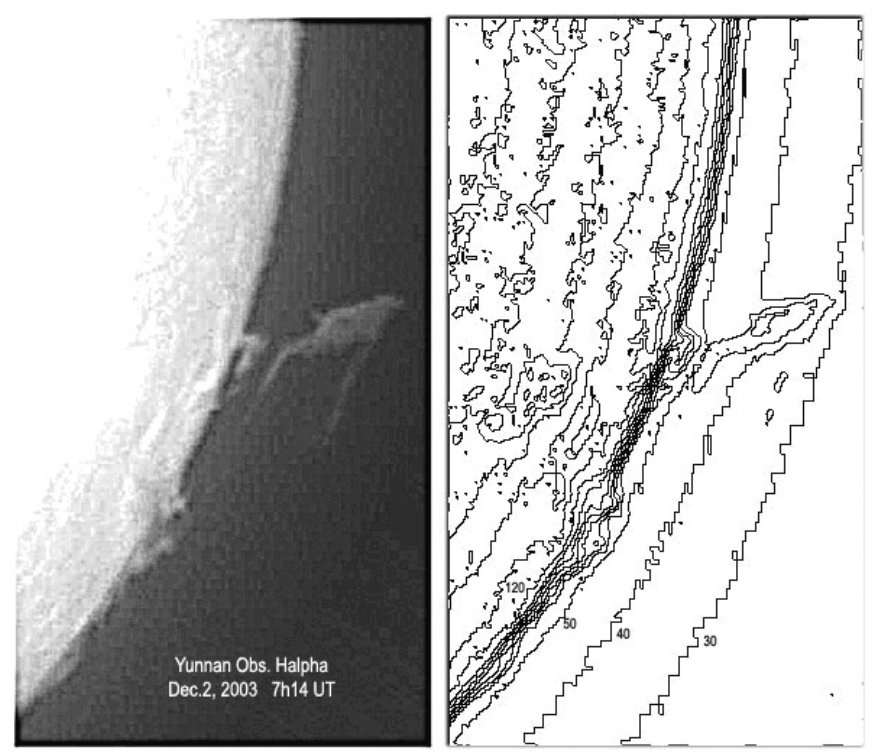

Fig. 2. Partial frame from an $\mathrm{H} \alpha$ filtergram taken before the eruption from the International $\mathrm{H} \alpha$ Network and, at left, the deduced set of calibrated isophotes used to evaluate the mass of the off-disk prominence. Note the levels of scattered parasitic light outside the solar disc (some typical intensities are shown near the bottom part).

to $1.35 R_{\odot}$ which could be related to the formation of an X-type neutral line in this region.

Gopalswamy et al. (2003) studied the temporal and spatial relations between the signatures of prominence eruptions detected in microwaves with the Nobeyama Radioheliograph and white-light CMEs. It was shown that in most of the studied events the prominence moved predominantly in the radial direction, with CMEs and EPs starting at nearly the same time. Jing et al. (2004) presented a statistical study of filament eruptions observed in $\mathrm{H} \alpha$ at the Big Bear Solar Observatory with other phenomena of solar activity. They found that most eruptions $(>50 \%)$ were associated with white-light CMEs, active region filament eruptions being more associated with flares than quiescent filament eruptions. Because of their close relationship to CMEs, EPs were regarded as one of the main sources of material in the mass balance of CMEs. Indeed, from the theoretical modeling point of view, the mass of the prominence could be an issue when considering the launch of the CME (Low et al. 2003) or, more generally, its associated magnetic structure (see Chen et al. 2006). Further out, there are strong indications that the core in the three-part CME structure is associated with a prominence but this relation is not direct; multi-wavelength observations are needed.

Evaluations of the EP mass are traditionally based on observations of the $\mathrm{H} \alpha$ absorption of the corresponding filament on the disk prior to eruption, where they are seen as dark objects. The destabilization of filaments appears as a sudden disappearance in $\mathrm{H} \alpha$ or a disparition brusque (DB) (Tandberg-Hanssen 1974; Soru-Escaut \& Mouradian 1990) which can be caused by an increase of the plasma ionization degree (thermal effect) or which can also be a result of Doppler-Fizeau effects (dynamical DB) including a darkening produced by rapid mass motion (Heinzel \& Rompolt 1987). With TRACE usually a large, high but faint $\sim 1$ MK loop moves outward before anything else happens.

The approach to estimating the hydrogen density in filaments from $\mathrm{H} \alpha$ absorption was developed by Mein et al. (1996) and Heinzel et al. (1999) who used the non-LTE cloud model 
with optical thickness, Doppler width and velocity as input parameters. Because the hydrogen ionization degree in the nonLTE model is a free parameter, the total mass of the filament cannot be determined unambiguously. When the prominence is seen in emission outside the disk, a more direct evaluation can be attempted using both narrow passband W-L coronagraphic measurements and $\mathrm{H} \alpha$ line-flux emission imaging (see Koutchmy \& Nikolsky 1981). To make filtergrams, a narrow passband Lyot filter is usually used and the data need additional calibrations to take into account the broader line profile.

This problem was partly overcome by combining $\mathrm{H} \alpha$ observations with observations of filaments in coronal EUV lines, where filaments are seen as dark objects on the background of the underlying corona, on the disk or even outside the disk. EUV observations of filaments were started with the Skylab mission and then continued by SOHO (EIT and CDS) and TRACE. Batchelor \& Schmahl (1994) interpreted the EUV absorption of cool objects as a continuum absorption of the coronal radiation by neutral $\mathrm{H}$, and by neutral and ionized He depending on the wavelength. Kucera et al. (1998) and Penn (2000) used CDS data for prominence diagnostics and obtained a column density of neutral hydrogen $\mathrm{HI}$ of $\sim 4 \times 10^{17}-10^{18} \mathrm{~cm}^{-2}$. Mein et al. (2001) have shown that a combination of $\mathrm{H} \alpha$, Ca II (8542 $\AA$ ) and TRACE $171 \AA$ data may yield a more self-consistent determination of the neutral hydrogen column density, of the electron density and of the electron temperature.

Heinzel et al. (2001) and Schmieder et al. (2003, 2004) noticed that filaments are more extended in EUV spectral lines with wavelengths below the hydrogen Lyman-continuum edge (912 $\AA$ ) than in $\mathrm{H} \alpha$ and they introduced the term "EUV filament channel" (EFC) to describe their observations. A spectroscopic model of the EUV absorption of filaments and prominences was suggested by Heinzel et al. (2003a,b), Anzer \& Heinzel (2005), and Schwartz et al. (2006). The method takes the absorption of EUV-line radiation by the Lyman continuum self-consistently into account, as well as the volume-blocking effect that is potentially important for coronal lines. A similar technique for deriving prominence mass from EUV absorption features was developed by Gilbert et al. (2005, 2006). However, when the EUV filament is seen as a prominence on the off-disk coronal background, often an edge-brightening around the prominence is observed, strongly suggesting that heated coronal material is concentrated at the periphery of the prominence. De Boer et al. (1998) analyzed the periphery of a prominence using transition region (TR) line emission and describes this enhanced emission with non-thermal broadenings and velocities reaching values up to $45 \mathrm{~km} \mathrm{~s}^{-1}$, which is considerably higher than what is observed using the profiles of cool lines inside the prominence. During the onset of our EP we observed a similar coronal edge brightening.

Here we report on a large W-limb event, which occurred on December 2, 2003, 9-14h UT, in the vicinity of the old multipolar region AR 10508 which was well observed earlier but was still on the disk at the time of the CME. We analyse the observations of the prominence eruption taken in $\mathrm{H} \alpha$ full-line images with the HACO coronagraph of the Pic du Midi Observatory (Romeuf et al. 2007), images of the surrounding corona in EUV with SOHO/EIT (Delaboudiniere et al. 1995), and CORONASF/SPIRIT telescopes (Zhitnik et al. 2002), and W-L images of the associated CME taken with the LASCO (SOHO) C2 and C3 coronagraphs. We discuss in detail the spatial and the temporal development of the eruption, evaluate the kinematics and especially the mass budget of the CME-related phenomenon and we propose a possible scenario of the event. This approach is quite similar to the early work of Plunkett et al. (2000) where a fast limb CME event with an EP was studied, although no flare was reported (possibly because the region was already beyond the limb). Here we pay special attention to the mass budget, including the measurement of the EUV dimming effect, and we propose a model applicable to this particular event based on a computed magnetic field context to describe the whole event, instead of proposing a universal 2.5D model.

\section{Observations}

\subsection{The context}

A group of three massive filaments was observed one week before the event by the Global High-Resolution $\mathrm{H} \alpha$ Network observatories (BBSO image in Fig. 1a) in the vicinity of NOAA AR 10508, which is one rotation after the famous AR 10486 that produced extremely powerful X17 flares on October 29 and X28 on November 4 during the preceding Carrington rotation. The largest filament (which we will examine further) located to the north of the center of the AR, was divided into two parts by a strong negative magnetic field patch seen with MDI (Fig. 1b). The filament was seen in all EUV wavelengths with EIT and with SPIRIT in the $175 \AA$ and $304 \AA$ bands as a dark feature. In coronal lines the filament was covered by overlaying hot loops of the active region. During a week, from November 25 to December 2, GOES observed more than 20 X-ray flares in this active region and the filament was not yet destabilized by the flares.

We estimate the geometrical parameters, intensities and derived physical values of two parts of the filament marked in Fig. 1b (top half) as $\mathrm{f} 1$ and $\mathrm{f} 2$, from its $\mathrm{H} \alpha$ image taken on November 26, 2003 at 17:57:25 UT. The data are shown in Table 1 (the thickness $D$ was not measured and was taken as equal to the average visible width). Taking into account that the measured intensity is the sum of partly absorbed background and foreground, we estimated the optical thickness in $\mathrm{H} \alpha$. Assuming the mean temperature of the filament body $(8000 \mathrm{~K})$ and following the approach of Heinzel et al. (2003a), we estimate $\tau_{912}$ which gives the neutral hydrogen density $n_{1}$, electron (H-ion) density $n_{\mathrm{e}}$ and the total hydrogen density $n_{\mathrm{H}}$. This gives the mass of both parts of the $\mathrm{H} \alpha$ filament $M_{\mathrm{H} \alpha}=1.3 \times 10^{15} \mathrm{~g}$. According to Aulanier \& Schmieder (2002), and Heinzel et al. (2003b), the EFC defined above contains an additional mass of $50-100 \%$ of the $\mathrm{H} \alpha$ filament mass, although we are not sure we see the EFC in our case, see Fig. 1. The total mass of the filament material $(\mathrm{H} \alpha+\mathrm{EFC})$ is then estimated as $M_{\mathrm{F}}=(2.3 \pm .3) \times 10^{15} \mathrm{~g}$.

\subsection{The erupting prominence and the flare}

On December 1 all day and on December 2 before 09:00 UT, a filament previously studied on the disk was observed on the west limb in $\mathrm{H} \alpha$ and in EUV lines (with SPIRIT and EIT) as a quiescent prominence with a center of mass lying at a height of $\sim 63 \mathrm{Mm}$. Later it became an erupting prominence (EP) that we studied in detail. After 09:10 UT the prominence was observed in the full $\mathrm{H} \alpha$ line with the HACO (H-Alpha COronagraph) imager at Pic du Midi observatory (see Romeuf et al. 2007, for an exhaustive description of HACO observations) with a cadence of $15 \mathrm{~s}$. Above the limb a prominence is seen in $\mathrm{H} \alpha$ as a luminous object because of its large opacity to UV and EUV radiation from the corona, the TR and the chromosphere (Heinzel \& Rompolt 1987). HACO collects the full flux produced by the $\mathrm{H} \alpha$ line (spontaneous transition 3-2 after level 3 is excited), 

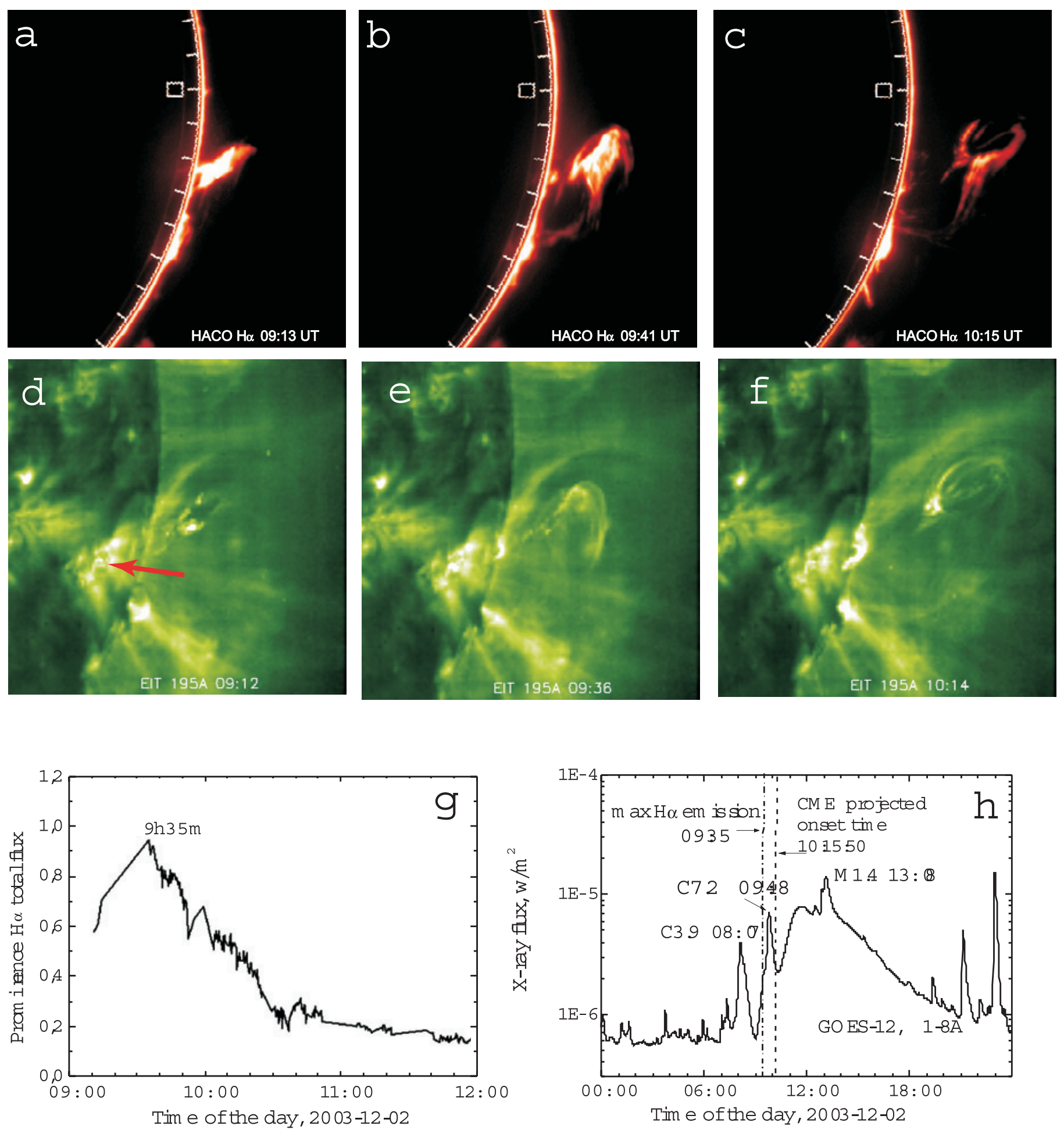

Fig. 3. Active phase of the prominence eruption seen with HACO in $\mathrm{H} \alpha \mathbf{a}$ )-c) and in EIT $195 \AA$ d) $-\mathbf{f}$ ). The arrow marks the possible place of emergence of the magnetic loop. In the bottom panels, the time variations of the total flux in $\mathrm{H} \alpha$ measured with HACO g) and, over the full day time interval, in X-rays 1-8 Å measured with GOES-12 h).

Table 1. Geometrical and physical parameters of the filament.

\begin{tabular}{llllllllll}
\hline \hline Filament part & $D, \mathrm{~cm}$ & $S, \mathrm{~cm}^{2}$ & $V, \mathrm{~cm}^{3}$ & $\tau_{\mathrm{H} \alpha}$ & $\tau_{912}$ & $n_{1}, \mathrm{~cm}^{-3}$ & $n_{\mathrm{e}}, \mathrm{cm}^{-3}$ & $n_{\mathrm{H}}, \mathrm{cm}^{-3}$ & $M_{\mathrm{H} \alpha}, \mathrm{g}$ \\
\hline $\mathrm{f} 1$ & $1.1 \times 10^{9}$ & $2.2 \times 10^{19}$ & $2.2 \times 10^{28}$ & 0.48 & 49 & $7.9 \times 10^{9}$ & $1.2 \times 10^{10}$ & $2.0 \times 10^{10}$ & $1.0 \times 10^{15}$ \\
$\mathrm{f} 2$ & $1.1 \times 10^{9}$ & $3.9 \times 10^{18}$ & $3.9 \times 10^{27}$ & 0.85 & 96 & $1.5 \times 10^{10}$ & $1.6 \times 10^{10}$ & $3.1 \times 10^{10}$ & $2.9 \times 10^{14}$ \\
\hline
\end{tabular}






Fig. 4. Temporal variations in relative units of i) the full $\mathrm{H} \alpha$ line flux, ii) the effective surface area of the prominence (inside a closed contour drawn at half intensity) and iii) the computed heights of the center of gravity and of the upper edge of the prominence; after 10:30 UT the values are less meaningful because the intensities of the EP drastically drop. The phase shifts of the temporal variations of these different parameters are illustrated.

independently of the dynamic state of the emitting elements (see Fig. 3) thanks to the broad width $(0.25 \mathrm{~nm})$ of the interference filter used by this instrument. This is in contrast to most instruments taking $\mathrm{H} \alpha$ filtergrams (see Fig. 2) which are affected by the Doppler shifts invariably occurring during the onset of an EP. In addition the level of scattered parasitic light is greatly reduced in HACO, compared to using a filtergram (see Figs. 3a-c).

At 09:13 UT we observed a new bright small expanding coronal loop emerging in the center of AR 10508 (indicated by an arrow in Fig. 3d), "striking" the prominence and destabilizing it. Figures $3 \mathrm{a}-\mathrm{f}$ show the most important stages of this process in $\mathrm{H} \alpha$ (HACO) and using EIT $195 \AA$ observations. EUV images synchronously show the expanding loop and increased heating of the gas around the prominence body (also called the periphery, see de Boer et al. 1998), which possibly corresponds to a part of the EUV filament channel. At the same time the brightness of the prominence body increased in $\mathrm{H} \alpha$ to its maximum value (at 09:35 UT, see Fig. 4). This is possibly due to the growth of turbulence inside, which would then produce a Doppler-brightening effect affecting its small elements (threads), as Gontikakis et al. (1997) showed using a detailed calculation for the case of an EP. Following these authors, the full flux in $\mathrm{H} \alpha$ could be magnified by a factor of 2 to 3 , which corresponds to what we observed in the body of the prominence for a short time, with turbulent velocities which keep the corresponding shifted line profiles still well within the pass-band of the filter, provided their transverse components do not exceed $70 \mathrm{~km} \mathrm{~s}^{-1}$.

We looked into the details of these short brightenings using a simultaneous histogram analysis of each consecutive image taken at high rate (15 s cadence; not shown here), keeping in mind all possible variations of instrumental and atmospheric origin (seeing), to conclude that the prominence was really agitated "inside". But the details of this analysis are beyond the scope of this work and we present here only the summary curves shown in Fig. 4 with some small scale rapid variations which could partly be produced by seeing effects. We also note that the increase of $\mathrm{H} \alpha$ flux is difficult to attribute to a sudden increase of the density or of the pressure inside the prominence: neither the morphology nor the kinematics of the EP suggests this.

An increase of the turbulence inside the body of the EP giving an increase in $\mathrm{H} \alpha$ emission, preceding any acceleration upward (or downward) of the main parts of the EP (see Fig. 4), is a novel feature which tells us something about the heating processes of the EP before its launch. The measured behavior of the variations of the effective surface (Fig. 4) of the prominence is an additional argument in favor of the "turbulent" scenario because of the observed phase shift between the different curves: i) variation of fluxes, ii) variations of the effective surfaces and iii) the motion of the center of gravity of the prominence, plotted in Fig. 4. One possibility to explain this increase of turbulence inside the prominence is to consider processes leading to short period ( 3 to $6 \mathrm{~min}$ ) oscillations which would put its threads into rapid transverse motion. Subsequently, a significant rate of dissipation (thermal heating due to wave dissipation, for example) would then increase the ionization ratio and ultimately, will make the EP disappear in $\mathrm{H} \alpha$ and make it then appear in emission in the hotter EUV lines. An argument in favor of this process comes from the examination of the TRACE movies of filaments seen in absorption in the $171 \AA$ coronal channel. They illustrate several cases of agitation phenomena occurring before the activation (rising) leading to ionization and eruption (see the filament movies produced by the Lockheed group; Title, 2006, personal communication) of a filament/prominence, although the limited field of view does not always permit a full evaluation of dynamical events linked to a CME. In TRACE, bright emission (Fe IX/X and Fe XII) is usually seen intermixed with the dark absorption during the eruption, not a long time before (see also Herant et al. 1991, Figs. 4-6).

After 09:41 UT the brightness of our prominence decreased in $\mathrm{H} \alpha$ and increased in EUV due to heating and ionization processes and it started to rapidly move outward, with the hottest part directed towards the Sun. At 09:48 UT the GOES X-ray intensity reached its maximum value corresponding to a $\mathrm{C} 7.2$ flare. Figures $3 \mathrm{e}, \mathrm{g}$ show the light curves of the total flux in $\mathrm{H} \alpha$, measured with HACO and in X-rays (1-8 A) measured with GOES 12.

\subsection{The CME in $W-L$}

The CME associated with the eruption appeared in the LASCO C2 field of view after 10:50 UT. Before this time (e.g. at 10:26 UT) a weak streamer was seen nearby at $225^{\circ}$. As a result of the CME development, the streamer at the periphery of the CME was deflected to the South and its brightness gradually increased with time. The CME had a typical three-part structure a frontal bright loop, a dark cavity and a bright core (see Fig. 5). The shape of the core resembled the shape of the heated EFC in $195 \AA$ (Fig. 3f). After 11:06 UT the frontal structure and, 20 min later, the core of the CME left the $\mathrm{C} 2$ field of view. In C3 the full CME was seen only in one frame at 12:18 UT.

According to the LASCO catalogue, the CME (frontal structure) moved in the $\mathrm{C} 2$ and $\mathrm{C} 3$ from 4 to $25 R_{\odot}$ with a nearly constant velocity of $1393 \mathrm{~km} \mathrm{~s}^{-1}$. At $6 R_{\odot}$ the core moved with a velocity of $942 \mathrm{~km} \mathrm{~s}^{-1}$. The points corresponding to the core in the height-time graph match well the same line as the last point for the prominence, which suggests a close relationship. The activation and the motion of the prominence started at least half an hour before the CME onset determined from a linear interpolation of the LASCO points.

\section{Mass loading and kinematics of the eruption and the CME}

The first component to consider is the mass directly injected or loaded by the EP and its surroundings. We discussed in Sect. 2.1 

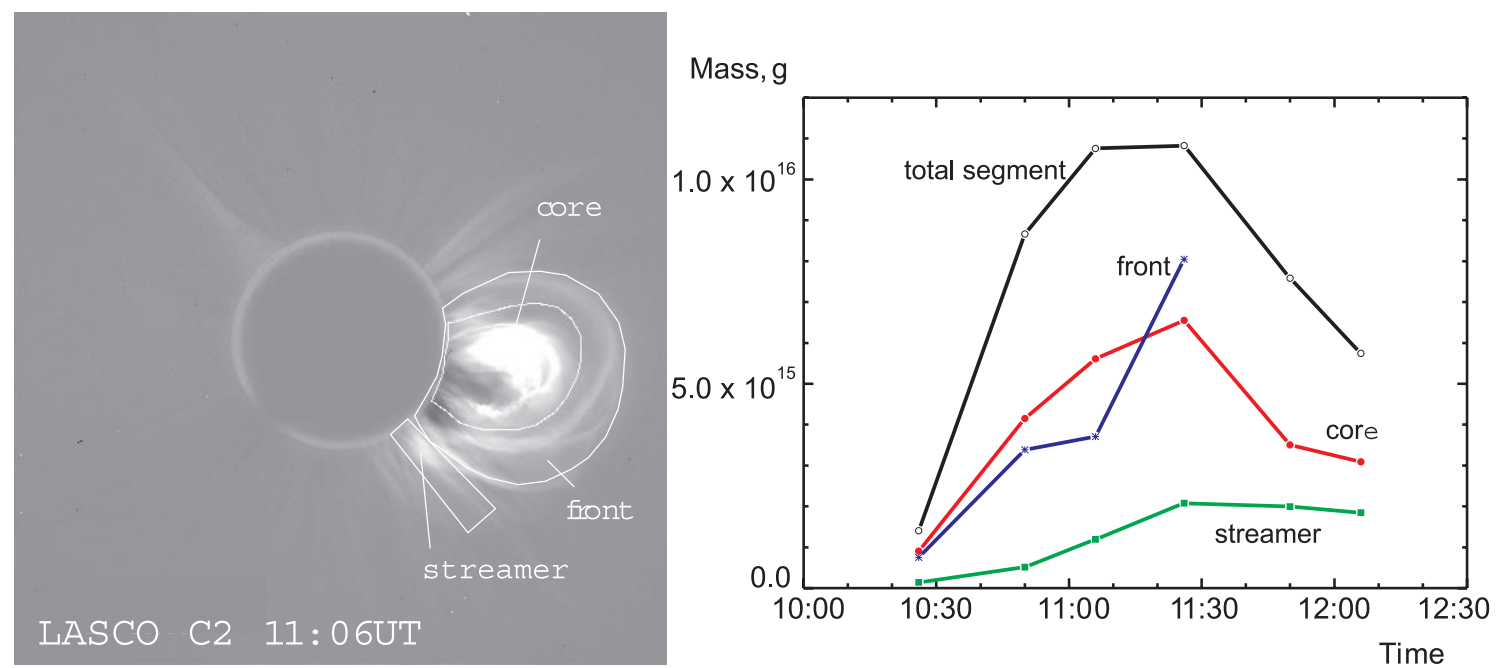

Fig. 5. Estimation of the CME mass from LASCO C2 images: (left - the integrated regions corresponding to the frontal structure, the core of the $\mathrm{CME}$ and to the streamer; (right) - the equivalent mass corresponding to the shown integrated intensities.
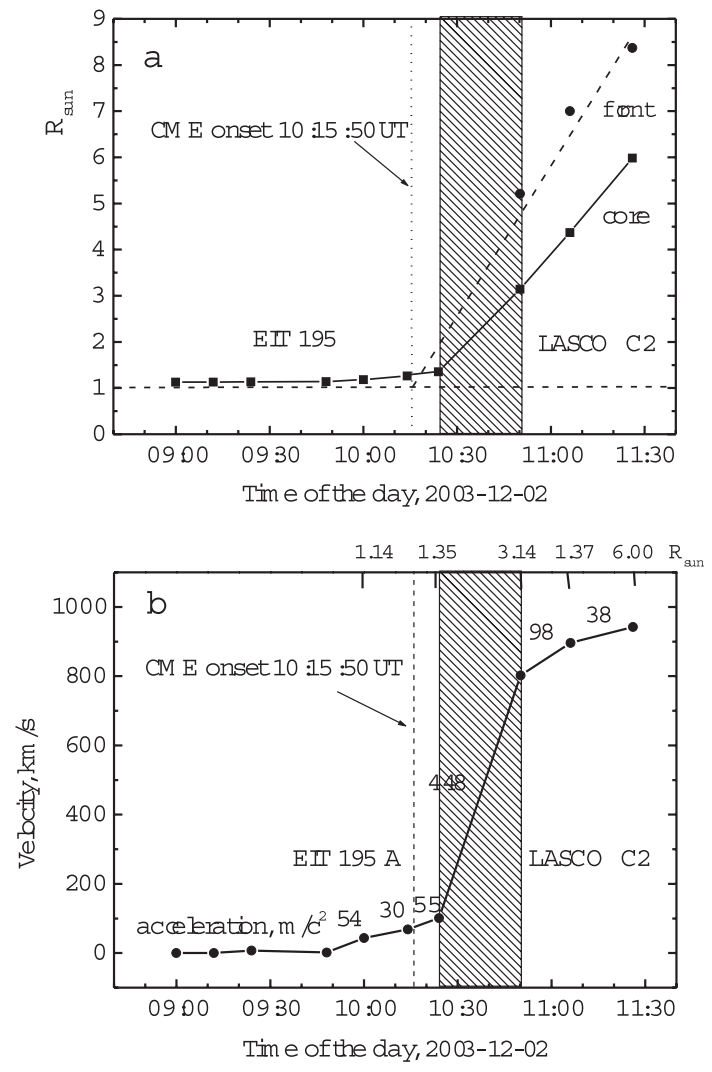

Fig. 6. Height-time profile of the prominence, the frontal structure and the core of the CME a); profile of the velocity and acceleration of the CME core b). The shaded area corresponds to the time interval where the eruptive material was out of fields of view of the instruments.

the determination of the mass of the $\mathrm{H} \alpha$ filament which was observed well before in the active region underlying the event (Fig. 1) and evaluated its mass, including its immediate surrounding, as $(2.3 \pm .3) \times 10^{15} \mathrm{~g}$ (this value possibly may be doubled due to turbulence).

We used several methods to evaluate the more relevant bulk mass of the prominence becoming the EP just prior to its launch (Figs. 2 and 3a). The effective surface area of the prominence is easy to measure. Then, we compared its average intensity and morphology with those of typical prominences that were measured with the aim of determining typical masses of prominences with the large $52 \mathrm{~cm}$ aperture coronagraph of the high altitude station near Kislovodsk. Narrow pass-band filters $(F W H M$ of $.5 \mathrm{~nm})$ in W-L (a continuum window near $607.3 \mathrm{~nm}$ ) and around $\mathrm{H} \alpha$ were used to perform a photometric analysis (Koutchmy \& Nikolsky 1981). We found a typical electron density integrated along the l.o.s. (or "column" density) of the order of $2.5 \times 10^{38} \mathrm{~cm}^{-2}$. Assuming an ionisation rate of $50 \%$ over the $10^{3} \mathrm{Mm}^{2}$ effective surface we have a mass of $1.2 \times 10^{15} \mathrm{~g}$. Inside the prominence the ionisation ratio could be lower and the estimated mass of the prominence will increase by a factor of 2 to 4 .

The second method we used takes advantage of intensity measurements done on the filtergram shown in Fig. 2, by comparing the isophote intensity levels over the prominence image with the simultaneously observed disk intensities. We then can obtain the integrated 1.o.s. amount of plasma with neutral hydrogen in level 2, and then assumptions have to be made concerning the thermal equilibrium among the levels to determine the abundance of $\mathrm{H}$ I at ground level 1, where densities are several orders of magnitude higher, etc. At local thermo-dynamical equilibrium (LTE), it will depend on the excitation temperature (Gouttebroze et al. 1993) which we take to be of the order of $6000 \mathrm{~K}$. The deduced column density from the averaged values of $\mathrm{H} \alpha$ fluxes of Fig. 2 gives an overall mass, taking into account the extension shown by isophotes, quite close to the mass deduced from W-L coronagraphic observations, when a $50 \%$ ionisation ratio is assumed (see also Koutchmy \& Nikolsky 1981). It could mean that this assumption is not too far from reality. However, the ionisation ratio is a matter of discussion: it is certainly not uniform inside the prominence (and even across the section of a single thread) and at least at the periphery where $\mathrm{H} \alpha$ emission disappears, the ratio is close to $100 \%$. Further, the assumption of LTE is certainly not satisfied, and finally the temperature is not uniform (see Wiehr et al. 2007). In this respect, what the EP region looks like using different EIT channels is important. The $304 \AA$ image of $\mathrm{He}+$, taken several hours before the eruption, shows the prominence in absorption but surrounded by rather strong emission; it is similar but weaker in the $171 \AA$ (Fe IX/X) channel. In the $284 \AA$ channel where the coronal temperature is much higher (Fe XV), it is not like this: although the prominence is also seen in absorption, no enhancement is seen at the periphery and the coronal emission is more extended. This tells us that the 
extended surrounding of the EP is an important part of the whole eruption and that the mass of a large part of this area has to be taken into account (see the discussion of the dimming effect below).

In view of the large dispersion found when trying to determine the mass of the prominence from the observed $\mathrm{H} \alpha$ flux (we do not discuss errors produced by using averaged values without going into the details of the optical thickness variations and variations of the profile of the line), we refrain from giving formulae and numerical results, aside from the rough estimates given above. Our conclusion is that more coronagraphic observations of prominences are needed, such as observing simultaneously the $\mathrm{H} \alpha$ flux and the $\mathrm{K} \mathrm{Ca}$ II flux to deduce a parameter sensitive to the ionisation ratio of HI. The method promoted in EUV by Gilbert et al. $(2005,2006)$ should also be developed (up to now, their sample of prominences gave masses not exceeding $10^{15} \mathrm{~g}$ ).

We also evaluated the mass of the CME using the LASCO images and the standard SolarSoft procedures, which include the allocation of regions of interest over which intensities are integrated. In each image we separately treated three regions, which correspond to the frontal structure, the core and the streamer (Fig. 5a). The plots of the calculated masses are presented in Fig. 5b.

The estimated values of masses corresponding to the time of the full CME expansion in the C2 field of view (11:26 UT) are: for the frontal structure $8 \times 10^{15} \mathrm{~g}$, for the core $6.6 \times 10^{15} \mathrm{~g}$, for the streamer $2 \times 10^{15} \mathrm{~g}$; for the total amount including all CME parts and the streamer $1.1 \times 10^{16} \mathrm{~g}$. The values for the frontal structure and for the core partly include the moving streamer component because it is difficult to clearly distinguish between them. A more realistic estimate is obtained after subtraction of the streamer mass from each component, which gives a mass of the frontal structure of $6 \times 10^{15} \mathrm{~g}$, for the core $4.6 \times 10^{15} \mathrm{~g}$. The sum of these values gives a full $\mathrm{CME}$ mass for $\mathrm{C} 2$ (without the streamer) of $10.6 \times 10^{15} \mathrm{~g}$. The total CME mass value for $\mathrm{C} 3 \mathrm{at}$ 13:42 UT $\left(R=25 R_{\odot}\right)$ is estimated as $1.5 \times 10^{16} \mathrm{~g}$, showing that the process of CME development effectively continued.

The total eruption/CME process can be roughly divided into three stages: activation, eruption of the prominence, and propagation of the CME in the LASCO field of view. Between the last moment where the prominence was seen in EUV or $\mathrm{H} \alpha$ (10:24 UT) and the first appearance of the CME in the LASCO C2 field of view (10:50 UT) there is a "grey zone" where the position of the erupting matter and the parameters of its evolution are not possible to determine.

We measured the positions of the moving prominence seen in absorption from the images taken with the EIT and the SPIRIT telescopes (from 09:00 UT to 10:24 UT) and combine it with the measured positions of the CME core in the LASCO C2 images. The height-time profiles of the prominence/core and frontal structure are shown in Fig. 6a, the derived values of velocity and acceleration of the core - in Fig. 6b. The dotted lines in these figures correspond to the projected CME onset time from the LASCO CME catalogue (http: //cdaw.gsfc . nasa. gov/CME_list) where it was derived from the height-time profile for the frontal structure using a linear approximation.

\section{EUV dimmings}

In general, dimmings (areas of temporarily reduced emission measure) in EUV or soft X-ray images appear at the places of the coronal plasma outflow during an eruption leading to a CME (e.g. Zhukov \& Auchere 2007). It is interpreted as a density depletion associated with the CME initiation
(Hudson \& Webb 1997; Zarro et al. 1999, and others). The procedure of image pre-processing and of selection of the dimming regions we used here was described in detail elsewhere (Chertok et al. 2004; Chertok \& Grechnev 2005; Slemzin et al. 2005, 2006). A detailed analysis of dimmings and their relationship with CMEs have been carried out in many papers (e.g. Harra \& Sterling 2001; Harrison et al. 2003; Grechnev et al. 2006, and so on). Maps of dimming regions we obtained in $195 \AA$ and $175 \AA$ are shown in Figs. 7a and b. Graphs in Fig. 7c display the light curves of the total dimming intensities relative to the total intensities in the original (not differenced) nominal images. The light curves for both cases are similar: they reached a maximum at $\sim 09: 30$ UT-10:00 UT before the CME onset, then decreased to a minimum at 11:30 UT-12:00 UT. The drop of intensity in the dimming area relative to the total solar intensity before the eruption is large: in $195 \AA$ it is about $7 \%$, in $175 \AA$ it is about $4.5 \%$. The spectral response functions of both bands are quite similar except that a small, pronounced hot component (10-15 MK) exists in the $195 \AA$ case.

We note that the beginning of the gradual dimming intensity decrease in $195 \AA$ (Fig. 7c) corresponds to the moment of the launch of the prominence (dash-dot line in Fig. 7c) and the maximum of intensity of the X-ray flare. If we assume that the EUV dimming is associated with the formation of the frontal structure, one can conclude that both the frontal structure and the core of the CME were initiated very close in time (within 5-10 min).

\section{Interpretation: the magnetic field structure and the filament equilibrium}

There is no doubt that the filament destabilization and its eruption were related to the properties and the changes of the magnetic field in the active region. We first note that the CME morphology (see Fig. 5) seems to fit one of those identified by Cremades \& Bothmer (2004), where the observed morphology seems to be consistent with what is expected for the neutral line location and the position of the eruption near the limb. However, the coronal magnetic field is not measured nor even qualitatively observed. Accordingly, this part of the work is mainly based on extrapolations made from the surface of the Sun and on guesses of the role of the magnetic field. The position of the active region, close to the limb on December 2, was favorable for measuring the plasma density distribution and the velocity of coronal structures but made it almost impossible to see the changes in the photospheric magnetic field. In order to guess the magnetic configuration in the erupting region we started from the time when the region was not far from the center of the disc on November 25.

We calculated the current-free coronal magnetic field ${ }^{1} \boldsymbol{B}$ in the vicinity of the filament using the photospheric magnetic field measurements from SOHO/MDI. As the region of interest was not large compared with the whole surface of the hemisphere, we considered the boundary as a flat surface restricted by the size of the active region containing the main magnetic concentrations. We used the well-known solution for half-space with a plane boundary:

$\boldsymbol{B}=\frac{1}{2 \pi} \iint_{S} \frac{B_{n}\left(x^{\prime}, y^{\prime}, 0\right) \boldsymbol{r}}{r^{3}} \mathrm{~d} x^{\prime} \mathrm{d} y^{\prime}$,

${ }^{1}$ Generally speaking, the presence of a non-potential flux rope is a necessary part of the model. A quite similar approach was recently attempted in the context of an active region (van Ballegooijen \& Mackay 2007). 

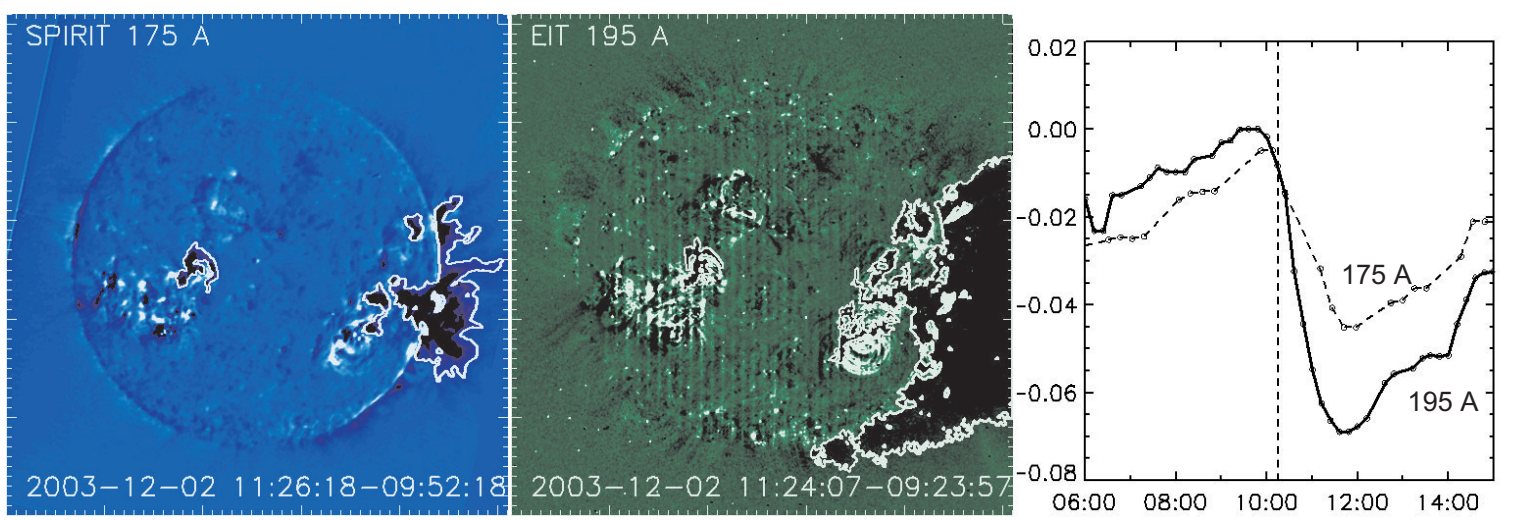

Fig. 7. Left and center panels - EUV dimmings in the SPIRIT $175 \AA$ and EIT $195 \AA$ difference images. Right panel - the total intensity in the dimming area relative to the total intensity of the nominal images. Dashed line corresponds to the projected LASCO CME onset (10:15:50 UT).

where $B_{\mathrm{n}}$ is the normal magnetic-field component on the plane $S$ and $\boldsymbol{r}$ is the radius vector from some point on the surface to a given point in the corona. Zagnetko et al. (2005) showed that the prominence material is mainly concentrated at the surface that passes through the apex of the field line arches which is a magnetic neutral surface. Recall that the systematic inclination of the bodies of large quiescent prominences at moderate latitudes toward the west, which had been widely described by d'Azambuja \& d'Azambuja (1948), was explained by the peculiarities of the structure of the large-scale magnetic fields. We calculated the coordinates of the neutral surface in AR 10508. Figure 8 shows the projection of the magnetic neutral surface, as it should look near the limb, calculated above the neutral line related to the studied filament up to the height of $150 \mathrm{Mm}$. We can compare the shape of a nearly semicircular loop lying in the neutral surface (Fig. 8, right) with the shape of the prominence at the early stage of the eruption (Figs. 3b,c) when the loop of the eruptive prominence roughly conserves its initial form. At equilibrium, the prominence loop should be located near the neutral surface, hence the front leg of the prominence should be curved and in the middle part nearly parallel to the solar surface, while the back leg should be more straight and tilted from the vertical direction at an angle of about $30^{\circ}$. This is qualitatively the same as what we see in Figs. 3b,c.

Then we found the limiting height of the filament equilibrium $h_{\mathrm{c}}$ by solving the equation (Filippov \& Den 2000, 2001)

$$
\left.\frac{\mathrm{d} B}{\mathrm{~d} h}\right|_{h_{\mathrm{c}}}=-\frac{B\left(h_{\mathrm{c}}\right)}{h_{\mathrm{c}}} .
$$

This parameter is derived from the vertical stability condition in the inverse polarity filament model (Van Tend \& Kuperus 1978; Molodensky \& Filippov 1987; Forbes \& Isenberg 1991) and reflects the scale height of the magnetic field of photospheric sources. We calculated the limiting height using the SOHO/MDI magnetograms of November 25, 27, and 29. The results for the two former days are very similar. Figure 9 shows the calculated neutral line at the height of $6 \mathrm{Mm}$, where the field is smoothed enough, overlaid on the photospheric magnetogram. The filament that erupts on December 2 is also shown as a white contour. In the middle part of the filament length, where its axis is rather straight, the limiting height is higher than the vertical size of our calculation domain $(150 \mathrm{Mm})$. Near the filament ends, the curvature of the neutral line increases. This means that the local scale of the photospheric magnetic field is smaller there. In accordance with the reduced field scale, the limiting height is lower and reaches $\sim 60 \mathrm{Mm}$. However, near the filament ends field lines are anchored in the photosphere and the magnetic
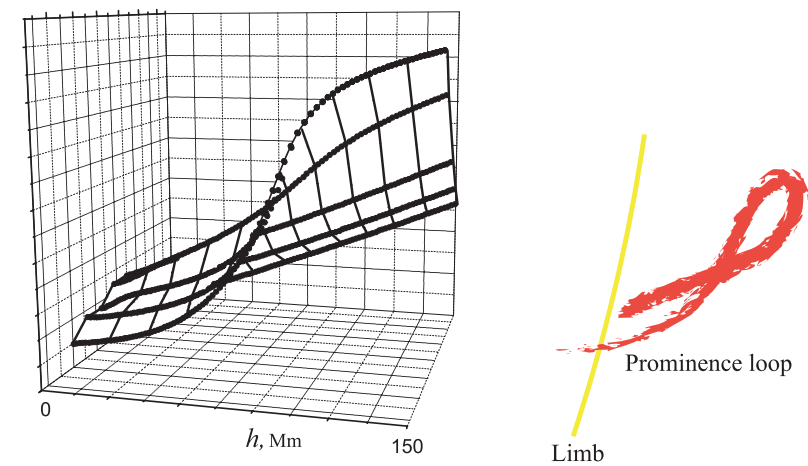

Fig. 8. Projection of the magnetic neutral surface in the AR 10508 on November 25, as it should look near the limb, calculated above the neutral line related to the eruptive prominence (left), and the side view of a loop lying in the neutral surface that models the eruptive prominence (right).

tension plays an important role, whereas Eq. (2) does not take into account the anchoring of the filament legs.

On November 29 the AR 10508 was close to the limb and the magnetic field measurements became unreliable. We then used a special procedure to obtain the component normal to the solar surface from the line-of-sight measurements (Den 2002). Calculations show a small lowering of the limiting height at the filament ends and a significant lowering in the middle of the filament down to $40 \mathrm{Mm}$. This value possibly reflects the tendency of a lowering of the limiting height; however, as has been mentioned, the initial photospheric magnetic field measurements in this region are not reliable.

Observations of the filament (and the corresponding prominence) near the limb on November 30 and December 1 demonstrate that the filament was stable up to a rather great height. On November 30 the height of the filament top can be estimated as $35 \mathrm{Mm}$, while on December 1 - as $50 \mathrm{Mm}$. The greatest height on December 2, before the filament begins to ascend, was $120 \mathrm{Mm}$. This value is smaller than the limiting height on 25-27 November (more than $150 \mathrm{Mm}$ ) but greater than the limiting height on November 29 (40 Mm, possibly underestimated). It is not unlikely that the height of the filament reaches a limiting value not long before the eruption, as has been shown for several tens of filaments by Filippov \& Zagnetko (2007). We believe that the rising of the filament, possibly due to the growth of an electric current (that we do not discuss here), within a flux rope containing the filament, up to the threshold of stability, is the main cause of the eruption. On the other hand, changes in the active region magnetic field can lower the threshold. When 


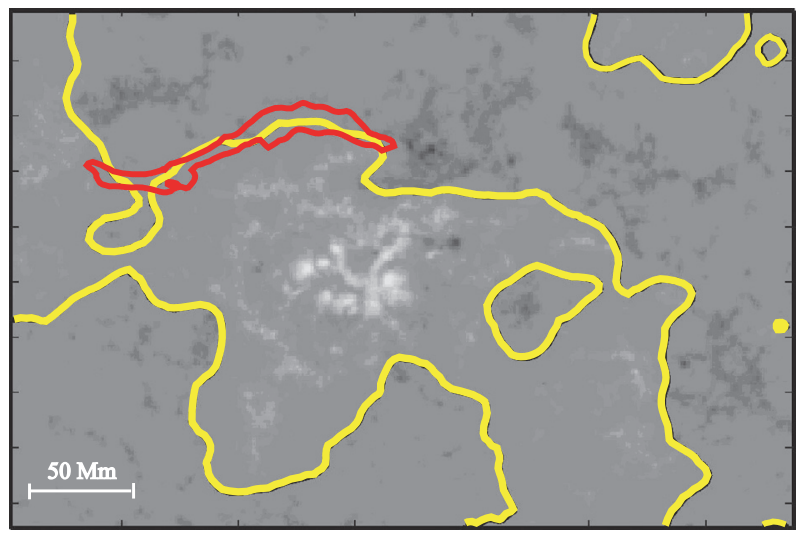

Fig. 9. The neutral line calculated at a level of $6 \mathrm{Mm}$ above the photosphere (yellow contours) overlaid on the photospheric MDI magnetogram obtained on 25 November 2003 at 19:12 UT within the area used as the boundary condition for coronal magnetic field calculations. The filament position taken from the BBSO $\mathrm{H} \alpha$ filtergram for 16:45 UT 25 November 2003 is shown as a red contour.

the height of the filament reaches the limiting height any small disturbance is able to initiate the beginning of the eruption.

The sequence of events observed on the west limb on December 2 between 07:30 UT and 11 UT can then be interpreted in the framework of a model of an erupting filament loop. We assume that the filament is the internal part of a twisted flux rope, which has the shape of a half torus, with both ends staying anchored in the chromosphere. The loop does not belong to a flat plane but lies in the curved neutral surface (Fig. 8). In accordance with the shape of the neutral surface, the fore leg of the arch is more curved. The arch apex was slowly ascending during several days before the eruption and reached a very high altitude by December 2 . We note that a quite similar schema has been recently proposed by Alexander et al. (2006) to describe what he called a "failed" filament eruption with a flare and RHESSI hard $\mathrm{X}$-ray emissions but no CME, so that we should still be cautious with the association of an EP and a CME. On the other hand, this scenario agrees rather well with the recent model of Chen et al. (2006), proposed to describe the CME-EP phenomenon.

The dark feature visible before 09 UT in EUV images, which coincides in space with the $\mathrm{H} \alpha$ prominence, overlaps the middle part and the far leg of the filament arch along the line of sight. But the activation and the ascent of the prominence began near its fore leg. The rising part becomes bright in EUV as is observed in many eruptive prominences (Filippov \& Koutchmy 2002), while the far part of the arch is still at rest. That is why we see rising bright features on the background of the rather static dark formation. This frontal part of the filament becomes clearly visible in the EIT images in emission at 09:48 UT and later. It is also clearly seen in the $\mathrm{H} \alpha$ images of the eruptive prominence as a curved feature (Figs. 3b,c).

The appearance of moving bright features within the body of an EP is consistent with the mechanism of heating of the prominence material proposed by Filippov \& Koutchmy (2002). The source of energy in this mechanism is the magnetic energy of the pre-eruptive magnetic configuration stored in the flux rope electric current and its interaction with the surrounding fields and plasma. The magnetic energy transforms into the macroscopic kinetic energy of the filament motion, including its threads. Part of this energy turns into heat through collisions inside the flux tube after an increase of the turbulence inside. A rather complex structure of the flux tube is necessary for this purpose. Some segments of the tube should have an upward concave form to provide the counter flow acceleration. We note that a typical twisting flux rope easily meets this requirement and that such twist was indeed observed in several instances (see e.g. Plunkett et al. 2000; Koutchmy et al. 2004; Sterling \& Moore 2004). It is also the requirement deduced from the latest theoretical analysis of the flux rope model proposed for CMEs by Chen et al. (2006).

\section{Conclusions}

We documented a large and fast coronal limb event, which occurred on December 2, 2003, in the interval 9-14h UT, in the vicinity of an old multi-polar region. Using observations obtained on the ground and in space with two EUV telescopes, an $\mathrm{H} \alpha$ coronagraph and the LASCO white-light coronagraphs, we analysed a spectacular dynamical phenomenon passing through different stages: a quiescent filament initially, then a prominence with EUV brightening at its periphery, its dynamical and thermal activation, a coronal eruption with a small flare and the launch of the eruptive prominence (EP) and finally the formation of a classical three-part fast CME. This CME fits rather well within the framework of the so-called "standard model", (see e.g. Lin 2004). Here we have added several features that we believe could shed some light on the whole process, emphasizing the mass transport processes related to the EP and its coronal surroundings.

From what we saw, the eruption process apparently started with the emergence of a new magnetic loop striking the prominence and destabilizing it. This led to a rapid heating of the gas around the prominence body, which spatially corresponds to a part of the EUV filament channel. The brightness of the body of the prominence in $\mathrm{H} \alpha$ at first increased to a maximum value (at 09:35 UT) possibly due to the growth of turbulence inside which produces a large Doppler brightening effect and, accordingly, magnifies the $\mathrm{H} \alpha$ flux without the need to increase the densities. This brightening even precedes the lifting of the cool prominence, which seems to be an important new feature to be taken into account in future work on CMEs. We note that this observation was made possible by the use of a coronagraph having a broad band filter and not a narrow band $\mathrm{H} \alpha$ filter as is usually done with a solar telescope to reject the parasitic light. After 09:41 UT the brightness decreased in $\mathrm{H} \alpha$ and increased in EUV, due to the enhanced ionization process and possibly a dissipation of the mechanical energy from waves or counter-flow motions. The prominence began to move outward at 09:48 UT, which coincides with the X-ray flare peak and would precede the CME extrapolated onset time.

A temporal analysis of the eruptive prominence motion and of the dimming intensity light curves has shown that both the frontal structure and the core of a CME were initiated simultaneously within 5-10 min around the peak of an X-ray flare.

Evaluations based on photometric data coming from the W-L coronagraphic coverage of the CME and from the photometric data provided by the $\mathrm{H} \alpha$ line-flux images of the prominence before its launch show that the total mass of the CME almost certainly surpasses the mass of the ejected prominence material seen in $\mathrm{H} \alpha$ before its eruption:

- The mass of the CME core is 2-2.5 times greater than the mass of the quiescent prominence, including the mass of an EUV channel (although taking into account possible turbulence this difference may vanish). This clearly shows that the mass of a fast CME is mainly due to the surrounding low corona swept out during the eruption, as confirmed by the large dimming effect we measured. This part of the CME is 
not treated in this paper but new ways already exist to consider it, e.g. Delannée et al. (2007).

- The dimming effect is observed over a large part of the corona, on the disk and also outside the disk when the line of sight integration reaches a 2 -fold increased value right above the limb with a corresponding increase of the dimming effect. The dimming is in phase with the onset of the CME. Approximately 5\% or more of the corona is depleted, neglecting the depletion of the mass of the corona at very low (TR) and/or very high coronal temperatures. The corona seen in the EIT $195 \AA$ channel should indeed correspond to the bulk of the mass because it corresponds to the most probable temperature of the corona, so that our estimate should not be far from the truth.

We also found that the eruption led to a spatial deflection and a brightening of the neighboring South-West streamer, which may give rise to an overestimate of the CME mass by $\sim 20 \%$. Further out toward both polar regions the deflections of structures such as extended plumes are clearly visible as a back and forth quasicoherent transverse motion. This phenomenon would need special study which is beyond the scope of this paper.

Finally our results suggest that greater attention should be paid to regions around the EP, bringing added justification to the theoretical studies trying to describe the behavior of the coronal magnetic field leading to CMEs. However only the field inside prominences can be easily measured with cool lines and the Hanle effect; an improved precision $\mathrm{H} \alpha$ coronagraph could routinely be used to perform such measurements at high speed.

Acknowledgements. The authors are grateful to O. G. Den for coronal magnetic field calculations, to J.-C. Vial for clarifications concerning the Doppler brightening effect, to G. Stellmacher for in-depth discussions on the determination of the prominence mass and the interpretation of prominence observations, to $\mathrm{C}$. Delannée, G. Lawrence for discussions on CMEs and to A. Zhukov for carefully looking at the 1st draft of the paper. This work was supported in part by the Russian Foundation for Basic Research (grants 06-02-16424 and 05-02-17415) and in part by the NATO CRG 940291 . SOHO is a space project of international cooperation between ESA and NASA and we specially thank the teams supporting the EIT and the LASCO observations for excellent work and providing their data to the community. S.K. also benefited from CNES in collaborating with the LASCO team in Marseille. The HACO ground-based coronal $\mathrm{H} \alpha$ observations are supported by the "Observateurs Associés" team sponsored by Fiducial LTD and by the "Observatoire Midi-Pyrénées". We are grateful to M. Audejean and J. Guignard for collecting the sequence used in this paper. We are also indebted to the international $\mathrm{H} \alpha$ Network for providing filtergrams to cover the chromospheric activity in 2003.

\section{References}

Alexander, D., Lin, R., \& Gilbert, H. R. 2006, ApJ, 653, 719

Aulanier, G., \& Schmieder, B. 2002, A\&A, 386, 1106

Anzer, U., \& Heinzel, P. 2005, ApJ, 622, 714

d'Azambuja, M., \& d'Azambuja, L. 1948, Ann. Obs. Paris, Meudon, V. 6, Fasc. VII

Bao, X., Zhang, H., Lin, J., \& Stenborg, G. A. 2007, A\&A, 463, 321

Batchelor, D. A., \& Schmahl, E. J. 1994, Solar Dynamic Phenomena and Solar Wind Consequences, ed. J. J. Hunt, ESA SP-373, 203

de Boer, C. R., Stellmacher, G., \& Wiehr, E. 1998, A\&A, 334, 280

Bothmer, V., \& Schwenn, R. 1994, Space Sci. Rev., 70, 215

Chen, J., Marqué, C., Vourlidas, A., Krall, J., \& Schuck, P. W. 2006, ApJ, 649, 452

Chertok, I. M., \& Grechnev, V. V. 2005, Sol. Phys., 229, 95
Chertok, I. M., Slemzin, V. A., Kuzin, S. V., et al. 2004, Astron. Rep., 48, 407 Cremades, H., \& Bothmer, V. 2004, A\&A, 422, 307

Delaboudiniere, J.-P., Artzner, G. E., Brunaud, J., et al. 1995, Sol. Phys., 162, 291

Delannée, C., Hochedez, J-F., \& Aulanier, G. 2007, A\&A, in press

Den, O. G. 2002, Astron. Lett., 28, 345

Filippov, B. P., \& Den, O. G. 2000, Astron. Lett., 26, 322

Filippov, B. P., \& Den, O. G. 2001, J. Geophys. Res., 106, 25177

Filippov, B., \& Koutchmy, S. 2002, Sol. Phys., 208, 283

Filippov, B., \& Zagnetko, A. 2007, J. Atmos. Sol.-Terr. Phys., in press

Forbes, T. G., \& Isenberg, P. A. 1991, ApJ, 373, 294

Gilbert, H. R., Holzer, T. E., Burkepile, J. T., \& Hundhausen, A. J. 2000, ApJ, 537,503

Gilbert, H. R., Falco, L. E., Holzer, T. E., \& MacQueen, R. M. 2005, ApJ, 618, 524

Gilbert, H. R., Falco, L. E., Holzer, T. E., \& MacQueen, R. M. 2006, ApJ, 641, 606

Gontikakis, C., Vial, J-C., \& Gouttebroze, P. 1997, A\&A, 325, 803

Gopalswamy, N. 2006, A\&A, 27, 243

Gopalswamy, N., Shimojo, M., Lu, W., et al. 2003, ApJ, 586, 562

Gouttebroze, P., Heinzel, P., \& Vial, J.-C. 1993, A\&AS, 99, 513

Grechnev, V. V., Chertok, I. M., Slemzin, V. A., et al. 2005, J. Geophys. Res., 110, A09S07

Harra, L. K., \& Sterling, A. C. 2001, ApJ, 561, 215

Harrison, R. A., Bryans, P., Simnett, G. M., \& Lyons, M. 2003, A\&A, 400, 1071

Heinzel, P., \& Rompolt, B. 1987, Sol. Phys., 110, 171

Heinzel, P., Mein, N., \& Mein, P. 1999, A\&A, 346, 322

Heinzel, P., Schmieder, B., \& Tziotziou, K. 2001, ApJ, 561, L223

Heinzel, P., Anzer, U., Schmieder, B., \& Schwarts, P. 2003a, ESA SP-535, 447

Heinzel, P., Anzer, U., \& Schmieder, B. 2003b, Sol. Phys., 216, 159

Herant, M., Pardo, F., Spiller, E., \& Golub, L. 1991, ApJ, 376, 797

Hudson, H. S., \& Webb, D. F. 1997, Coronal Mass Ejections, ed. N. Crooker,

J. Joselyn, \& J. Feynman (Washington, DC: AGU Geophysical Monographs 99), 27

Jing, J., Yurchyshyn, V. B., Yang, G., Xu, Y., \& Wang, H. 2004, ApJ, 614, 1054 Koutchmy, S., \& Nikolsky, G. M. 1981, Sov. Astron. Lett. 7, 102

Koutchmy, S., Baudin, F., Bocchialini, K., et al. 2004, A\&A, 420, 709

Kucera, T. A., Andretta, V., \& Poland, A. I. 1998, Sol. Phys., 183, 107

Lin, J. 2004, Sol. Phys., 222, 115

Low, B. C., Fong, B., \& Fan, Y. 2003, ApJ, 594, 1060

Mein, N., Mein, P., Heinzel, P., et al. 1996, A\&A, 309, 275

Mein, N., Schmieder, B., DeLuca, E. E., et al. 2001, ApJ, 556, 438

Molodensky, M. M., \& Filippov, B. P., 1987, Soviet Ast., 31, 564

Penn, M. J. 2000, Sol. Phys., 197, 313

Plunkett, S. P., Vourlidas, A., Simberova, S., et al. 2000, Sol. Phys., 194, 371

Romeuf, D., Meunier, N., Noens, J.-C., et al. 2007, A\&A, 462, 731

Schmieder, B., Tziotziou, K., \& Heinzel, P. 2003, A\&A, 401, 361

Schmieder, B., Lin, Y., Heinzel, P., \& Schwartz, P. 2004, Sol. Phys., 221, 297

Schwartz, P., Heinzel, P., Schmieder, B., \& Anzer, U. 2006, A\&A, 459, 651

Slemzin, V. A., Kuzin, S. V., Zhitnik, I. A., et al. 1995, Solar System Res., 39, 489

Slemzin, V. A., Grechnev, V. V., \& Kuzin, S. V. 2006, Solar Activity and its Magnetic Origin, Proc. ed. V. Bothmer, \& A. A. Hady (Cambridge: Cambridge University Press), IAU Symp., 233, 361

Soru-Escaut, I., \& Mouradian, Z. 1990, A\&A, 230, 474

Sterling, A. C., \& Moore, R. L. 2004, ApJ, 599, 1418

Tandberg-Hanssen, E. 1974, Solar Prominences (Dordrecht, Holland: D. Reidel Publ. Co.)

Van Tend, W., \& Kuperus, M. 1978, Sol. Phys. 59, 115

van Ballegooijen, A. A., \& Mackay, D. H. 2007, ApJ, 659, 1713

Vourlidas, A., Subramanian, P., Dere, K. P., \& Howard, R. A. 2000, ApJ, 534, 456

Wagner, W. J. 1984, ARA\&A, 22, 267

Wiehr, E., Stellmacher, G., \& Hirzberger, J. 2007, Sol. Phys., 240, 25

Zagnetko, A. M., Filippov, B. P., \& Den, O. G. 2005, Astron. Rep., 49, 425

Zarro, D. M., Sterling, A. C., Thompson, B. J., Hudson, H. S., \& Nitta, N. 1999, ApJ, 520, L139

Zhitnik, I. A., Bougaenko, O. I., Delaboudiniere, J.-P., et al. 2002, ESA SP-506,

Solar Variability: from Core to Outer Frontiers, 915

Zhukov, A. N., \& Auchère, F. 2007, A\&A, 427, 705 